\title{
Editorial
}

\section{Formal ontologies in manufacturing}

\author{
Emilio M. Sanfilippo ${ }^{\mathrm{a}, \mathrm{b}, *}$, Yoshinobu Kitamura ${ }^{\mathrm{c}}$ and Robert I.M. Young ${ }^{\mathrm{d}}$ \\ ${ }^{a}$ Le Studium, Loire Valley Institute for Advanced Studies, Orléans \& Tours, France \\ ${ }^{\mathrm{b}}$ CESR - Université de Tours, 59, rue Néricault-Destouches, 37020 Tours, France \\ E-mail: emilio.sanfilippo@univ-tours.fr \\ ${ }^{\mathrm{c}}$ College of Information Science \& Engineering, Ritsumeikan University, Japan \\ E-mail:y-kita@fc.ritsumei.ac.jp \\ ${ }^{\mathrm{d}}$ School of Mechanical, Electrical and Manufacturing Engineering, Loughborough University, UK \\ E-mail: ri.young@lboro.ac.uk
}

Since the early days of ontology engineering, manufacturing is one of the main areas where ontologies have traditionally been applied (Guarino et al., 1997; Uschold and Grüninger, 1996). The interest in ontologies has been motivated, first, by the massive exploitation of computer-based technologies in manufacturing organizations, which need to manage and share data in a robust way, and second, by the need to harmonize different terminologies to facilitate communication. The two motivations are strictly related, since shared terminologies and models are needed to enable computer systems to interact effectively. In addition, in the new landscape of Industry 4.0 (Lu, 2017), guided and informed by big data and machine learning, ontologies find their place to organize the data upon which learning algorithms run.

Looking at the literature on manufacturing ontologies, research efforts can be roughly classified in two broad segments: the first one aimed at establishing the conceptual and formal foundations of manufacturing ontologies (Bock et al., 2010; Borgo and Leitão, 2007; Grüninger, 2009; Usman et al., 2013), and the second one specifically focused on the use of ontologies in application systems (Colombo et al., 2007; Matsokis and Kiritsis, 2010; Perzylo et al., 2015; Tessier and Wang, 2013; Terkaj et al., 2012). Unfortunately, the two research lines have been co-existing for a long time with only little interaction. Theoretical studies have been left aside and the manufacturing community still lacks robust methodologies for ontology design. At the same time, software applications have not been used as testbed for formal ontologies and it is therefore hard for experts to exploit them. There is few reports on industrial deployment of software applications based on deeply-investigated ontologies (Kitamura et al., 2006). It is only recently that some efforts towards the interaction of the two research lines have been carried out. The Industrial Ontologies Foundry (IOF, 2019) is probably the most significant and ambitious example, although it is still too early to say whether it will succeed in supporting the multiple cross-functional interoperability requirements of manufacturing businesses and provide the ontologies, methodologies, and technologies that stakeholders will adopt.

\footnotetext{
*Corresponding author. E-mail: emiliosanfilippo@gmail.com (permanent address).
} 
Independently from the dichotomy between foundational and applied-oriented studies, the ontological representation of manufacturing knowledge has been widely investigated. Traditionally, emphasis has been given to the representation of product knowledge leading to ontologies about features, components, or products' qualities (Fiorentini et al., 2007; Imran and Young, 2015; Vegetti et al., 2011). In the 90's, some scholars proposed the use of mereologies and mereo-topologies to rigorously model domainspecific spatial relations for product knowledge representation (Borst and Akkermans, 1997; Guarino et al., 1997; Salustri, 1997; Simons and Dement, 1996). This research topic, which was left apart for some years, is getting a momentum nowadays probably under the pressure of advanced technologies that demand robust theories to organize product data (see the work of Aameri et al., 2019 in this Special Issue for further references). Surprisingly enough, less attention has been given to (the ontological understanding of) manufacturing processes. Despite many papers and the availability of ISO standards like STEP-NC (ISO 10303-238; ISO, 2003) and PSL (ISO 18629; Grüninger and Menzel, 2003), the formal and ontological characterization of manufacturing processes, including the resources used, consumed, or transformed during their execution, is a topic for open research. As a matter of fact, disagreements often arise on the very notion of manufacturing process, e.g., whether it covers only the creation (or refinement) of products, or it should be rather broadly understood to cover a larger spectrum of processes occurring in manufacturing organizations. The partial treatment of manufacturing process knowledge has some consequences for the integration of product and process knowledge, for which mature formal and ontological works are scarce. Finally, recall that the wide use of information systems in manufacturing contributed to the development of ontologies in disparate areas including industrial plants design or (re-)configuration (Kádár et al., 2013), maintenance (see the work of Karray et al., 2019 in this Special Issue), supply chain data management (Palmer et al., 2016), and product service systems (Shani et al., 2017), among others.

The requirements of manufacturing industry lead to many open research topics in manufacturing ontology that include (but are not limited to):

- Ontological modeling of manufacturing resources: in the context of Industry 4.0 and under the pressure of continuously evolving manufacturing methods like additive manufacturing, researchers are currently investigating the ontological representation of complex manufacturing resources like Cyber-Physical System (CPS; Horváth and Gerritsen, 2012). These are challenging to be represented and conceptualized not only because of the complex software-hardware structure they have, but also because of their agentive dimension. In order for ontologies to properly characterize CPS, the ontology of "traditional" manufacturing resources needs to be investigated beforehand. For example, it is common in manufacturing to assume an intuitive distinction between resources that execute the desired task such as cutting, polishing, or additive machines, from resources that support the execution of such tasks, e.g., fixtures, jigs, or gauges, among others, and resources that enable the functioning of other resources, e.g., lubricants, gasoline, or electric energy. The representation of CPS and other emergent Industry 4.0 resources would likely benefit of an ontological investigation of these and other similar distinctions.

- Ontological modeling of manufacturing systems: Industry 4.0 envisions the development of fully automated manufacturing environments where inter-connected (agentive) resources mutually interact by exchanging data and taking autonomous decisions on the task they are required to fulfill. These production systems are sometimes called Cyber-Physical Production Systems (CPPS; Monostori, 2014). From a modeling perspective, current research work addresses the use of ontologies to facilitate the exchange of data among the resources used in CPPS (Grangel-González et al., 
2016; Garetti et al., 2015). In our understanding, this focus on applications needs to be complemented with research efforts that address what it means for a CPPS to be a working environment where autonomous and agentive artificial resources interact between them and with humans. Some works towards the analysis of CPPS as socio-technical environments have been already carried out (see the work of Borgo and Sanfilippo, 2018 for further references); the results are however still preliminary and further efforts are required. Ontology designers may seek for contributions coming from areas where similar topics have been already investigated, e.g., social ontology, game theory, and multi-agents systems.

- Ontological modeling of manufacturing processes: a robust investigation on the ontological modeling of manufacturing processes is necessary. This includes (at least) 1) defining the very notion of manufacturing process; 2) identifying and distinguishing between different classes of manufacturing processes; 3 ) characterizing different ways in which objects (e.g., resources) participate in processes; 4) conceptualizing and modeling the relations between processes and plans, as well as between processes, plans, products, and design specifications.

- Ontological modeling of business notions relevant for manufacturing: it is common to describe the manufacturing world by emphasizing its business dimension. From this perspective, products are items that are sold for the economical benefit of companies (see the paper of Otte et al., 2019 in this Special Issue), processes are executed according to business policies and regulations, resources are owned by organizations, and manufacturing organizations themselves interact according to the business goals they are meant to achieve. Business-related notions have been left apart from manufacturing ontologies and their modeling is therefore needed.

- Adoption of foundational ontologies for manufacturing: the interest of the engineering community for foundational ontologies like the Basic Formal Ontology (BFO; Arp et al., 2015), the Unified Foundational Ontology (UFO; Guizzardi et al., 2015), or the Descriptive Ontology for Linguistic and Cognitive Engineering (DOLCE; Masolo et al., 2003), among others, is growing because of the need for well-defined and principled frameworks for knowledge and data management. By their very nature, foundational ontologies are complex systems whose technicalities are often hard to grasp by the average domain expert who likely lacks a background in formal logic or philosophical ontology (Stevens et al., 2019). In addition, only few works have attempted to measure the effectiveness of different foundational ontologies for practical needs such as data modeling or information systems interoperability (Keet, 2011; Stevens et al., 2019). A consequence is that the choice of adopting a certain foundational ontology is commonly motivated by concerns others than its conceptual or logical robustness. Efforts are required to avoid this and to help domain experts in selecting and properly using the foundational ontology that is best suited for their purposes. First, from a bottom-up perspective, robust case studies are needed to understand how efficient foundational ontologies are in satisfying manufacturing experts' requirements. Second, from a top-down perspective, ontologists need to define methodologies to support domain experts modeling tasks but also to help them in understanding the technical aspects of foundational ontologies. Third, different academic "schools of thoughts" should collaborate in transferring to stakeholders their knowledge and experience by promoting the methodologies they share rather than by competing for (possible) market benefits.

- Modeling languages: most of the ontologies currently available for the manufacturing domain are formalized in Semantic Web languages, the Web Ontology Language (OWL) foremost (Negri et al., 2016). This allows to rely on disparate technologies nowadays available (e.g., to store, query, or visualize data), as well as to reason over knowledge and data in a tractable manner. Notoriously, 
however, the expressivity of Semantic Web languages is limited and modelers often need to come with ad hoc workarounds, e.g., when ternary relations are needed. Alternative approaches have been explored within the manufacturing community but they have received only scarce attention (Grüninger and Katsumi, 2012; Imran and Young, 2015). We think that the research community would benefit from the investigation of modeling frameworks and languages other than Semantic Web languages to face the challenges of ontology modeling in manufacturing. Some inputs may come from the use of the Distributed Modeling Language (DOL; Mossakowski et al., 2015), logic programming approaches based on, e.g., Prolog (Lloyd, 1993) or Answer Set Programming (ASP; Lifschitz, 2008), or Common Logic based knowledge management systems (Grüninger and Katsumi, 2012; Imran and Young, 2015).

This list is not meant to exhaust the range of research topics to be addressed in manufacturing ontology. It is indeed likely the case that the emergence of new conceptual paradigms about the industrial world and the development of advanced computer-based technologies will enrich the research agenda. However, fundamental questions about the nature of the basic entities found in manufacturing ontologies (e.g., products, resources, processes, production systems, etc.) cannot and should not be dismissed. In addition, there are many practical issues that constrain manufacturing business competitiveness that can benefit from ontological solutions. Key amongst these are (i) interoperability across multi-domain, multi-business and multi-systems, (ii) software environments that support the rapid dynamic change requirements of businesses, (iii) minimizing the cost of developing business specific knowledge environments through the re-use of standardized ontologies, (iv) manufacturing knowledge maintenance methods as businesses advance their manufacturing understanding.

This Special Issue on Applied Ontology with title Formal Ontologies in Manufacturing has been thought to address foundational issues in manufacturing ontology but also to provide technical insights about ontology-based applications. Many journals in the manufacturing domain recurrently publish papers on ontologies, and the Semantic Web journal and the Journal of Engineering Design are about to publish special issues about ontologies in and for industry and design. On our side, the choice of editing this Special Issue on Applied Ontology depended on at least three reasons. First, Applied Ontology has historically adopted a strongly interdisciplinary approach, which is fundamental in our understanding to characterize a complex domain like manufacturing. Second, the journal emphasizes the need for principled approaches to guide the development of ontologies. Third, Applied Ontology welcomes papers where ontologies are represented in expressive formal languages. Our aim was therefore to collect research papers presenting challenging modeling problems concerning knowledge representation in manufacturing and providing mature ontological analysis and formal models.

The papers submitted for the Special Issue, out of which three are hereby published, spanned across the entire manufacturing domain, from formal theories to represent parthood relations for assembly, to manufacturing types modeling, agent-based systems for Industry 4.0, core manufacturing ontologies, the modeling of product variability, etc.

Aameri et al. (2019) present the Assembly Ontology for generative design: automatic generation of feasible design solutions based on given design goals and constraints. The ontology specifies connection, parthood, and shapes in mechanical assemblies for logical expressions of such qualitative constraints. The ontology extends the theory of Ground Mereotopology (MT) and combines it with a qualitative shape ontology based on the Hilbert's axiomatic theory of geometry. The paper describes its formal axiomatization and demonstrates its application in axiomatic representation of suspension systems. With respect to the research challenges mentioned above, this paper contributes to the formal treatment of engineering knowledge in languages other than OWL. Also, it contributes to the exploitation of theories 
of formal ontology like MT for engineering modeling purposes. In our understanding, the paper presents a promising research contribution to support both the representation of and reasoning over assembly structures in a principled way.

Karray et al. (2019) introduces the ROMAIN ontology for knowledge representation and data modeling in the manufacturing maintenance domain. The ontology builds on the BFO in order to reuse its upper-level modeling elements and enable data and applications interoperability with BFO-aligned ontologies. BFO was selected as reference upper-level because its successful use for interoperability has been already extensively documented. To validate ROMAIN against experts' knowledge, the paper presents a case study where the ontology is used to organize and retrieve maintenance data. From a more general perspective, this contribution is a step forward in the ontological modeling of maintenance knowledge; it also addresses challenging issues that researchers will need to face to foster the adoption of ontologies for maintenance. In addition, by relying on the BFO, the paper shows the willingness of the engineering community not only in using upper-level ontologies to facilitate ontology development or data/applications interoperability but also in analyzing domain knowledge with respect to formal ontology theories (e.g., theory of objects, qualities, processes, dependence, mereology, etc.).

Otte et al. (2019) present a suite of modular ontologies called the Product Life Cycle (PLC) ontologies for data system interoperability in the manufacturing industry. The ontology relies on both the BFO and the suite of mid-level ontologies called the Common Core Ontology (CCO). The paper discusses definitions of fundamental concepts in the product life cycle such as 'product', 'commodities', 'is input of', and 'provision of a service' based on the definitions in BFO. With respect to general research challenges, the paper is a contribution towards the definition of a core ontology for manufacturing based on a foundational ontology. Also, it presents an analysis of domain notions with respect to both engineering and economics, which - as previously said - is relevant to characterize the business dimension of manufacturing.

We hope that this Special Issue will contribute to stimulate the research community in digging into the ontological modeling of manufacturing knowledge with an open-minded attitude from both ontologists towards manufacturing and manufacturing experts towards formal ontology.

\section{References}

Aameri, B., Cheong, H. \& Beck, J.C. (2019). Towards an ontology for generative design of mechanical assemblies. Applied Ontology, 14(2), 127-153.

Arp, R., Smith, B. \& Spear, A.D. (2015). Building Ontologies with Basic Formal Ontology. MIT Press.

Bock, C., Zha, X., Suh, H.W. \& Lee, J.H. (2010). Ontological product modeling for collaborative design. Advanced Engineering Informatics, 24(4), 510-524. doi:10.1016/j.aei.2010.06.011.

Borgo, S. \& Leitão, P. (2007). Foundations for a core ontology of manufacturing. In R. Sharman, R. Kishore and R. Ramesh (Eds.), Ontologies. Integrated Series in Information Systems (Vol. 14, pp. 751-775). Boston, MA: Springer. doi:10.1007/ 978-0-387-37022-4_27.

Borgo, S. \& Sanfilippo, E.M. Components and interactions: Paving the way to model agent-based cyber-physical social systems. In Proceedings of TMCE 2018.

Borst, P. \& Akkermans, H. (1997). An ontology approach to product disassembly. In E. Plaza and R. Benjamins (Eds.), Knowledge Acquisition, Modeling and Management. EKAW 1997. Lecture Notes in Computer Science (Lecture Notes in Artificial Intelligence) (Vol. 1319, pp. 33-48). Berlin, Heidelberg: Springer.

Colombo, G., Mosca, A. \& Sartori, F. (2007). Towards the design of intelligent CAD systems: An ontological approach. Advanced Engineering Informatics, 21(2), 153-168. doi:10.1016/j.aei.2006.11.003.

Fiorentini, X., Gambino, I., Liang, V.C., Rachuri, S., Mani, M., Nistir, C.B., .. \& \& Turner, J.M. (2007). An ontology for assembly representation. NIST report 7436.

Garetti, M., Fumagalli, L. \& Negri, E. (2015). Role of ontologies for CPS implementation in manufacturing. Management and Production Engineering Review, 6(4), 26-32. doi:10.1515/mper-2015-0033. 
Grangel-González, I., Halilaj, L., Coskun, G., Auer, S., Collarana, D. \& Hoffmeister, M. (2016). Towards a semantic administrative shell for Industry 4.0 components. In Semantic Computing (ICSC), 2016 IEEE Tenth International Conference on (pp. 230-237). doi:10.1109/ICSC.2016.58.

Grüninger, M. (2009). The ontological stance for a manufacturing scenario. Journal of Cases on Information Technology (JCIT), 11(4), 1-25. doi:10.4018/jcit.2009072101.

Grüninger, M. \& Katsumi, M. (2012). Specifying ontology design patterns with an ontology repository. In Proceedings of the 3rd International Conference on Ontology Patterns. CEUR-WS (Vol. 929).

Grüninger, M. \& Menzel, C. (2003). The process specification language (PSL) theory and applications. AI Magazine, 24(3), 63-74.

Guarino, N., Borgo, S. \& Masolo, C. (1997). Logical modelling of product knowledge: Towards a well-founded semantics for STEP. In Proceedings of European Conference on Product Data Technology (pp. 183-190).

Guizzardi, G., Wagner, G., Almeida, J.P.A. \& Guizzardi, R.S. (2015). Towards ontological foundations for conceptual modeling: The unified foundational ontology (UFO) story. Applied Ontology, 10(3-4), 259-271. doi:10.3233/AO-150157.

Horváth, I. \& Gerritsen, B.H. (2012). Cyber-physical systems: Concepts, technologies and implementation principles. In Proceedings of TMCE (Vol. 1, pp. 7-11).

Imran, M. \& Young, B. (2015). The application of common logic based formal ontologies to assembly knowledge sharing. Journal of Intelligent Manufacturing, 26(1), 139-158. doi:10.1007/s10845-013-0768-4.

IOF (2019). Industrial Ontologies Foundry. Retrieved on April 2, 2018. Available at: https://www.industrialontologies.org/.

ISO (2003). Industrial automation systems and integration - Physical device control - Data model for computerized numerical controllers. Part 1: Overview and fundamental principles.

Kádár, B., Terkaj, W. \& Sacco, M. (2013). Semantic virtual factory supporting interoperable modelling and evaluation of production systems. CIRP Annals - Manufacturing Technology, 62(1), 443-446. doi:10.1016/j.cirp.2013.03.045.

Karray, M.H., Ameri, F., Hodkiewicz, M. \& Louge, T. (2019). ROMAIN: Towards a BFO compliant reference ontology for industrial maintenance. Applied Ontology, 14(2), 155-177.

Keet, C.M. (2011). The use of foundational ontologies in ontology development: An empirical assessment. In The Semantic Web: Research and Applications - 8th Extended Semantic Web Conference, ESWC 2011, Heraklion, Crete, Greece, Proceedings, Part I (pp. 321-335).

Kitamura, Y., Koji, Y. \& Mizoguchi, R. (2006). An ontological model of device function: Industrial deployment and lessons learned. Applied Ontology, 1(3/4), 237-262.

Lifschitz, V. (2008). What is answer set programming? In AAAI (Vol. 8, pp. 1594-1597).

Lloyd, J.W. (1993). Foundations of Logic Programming. Springer.

Lu, Y. (2017). Industry 4.0: A survey on technologies, applications and open research issues. Journal of Industrial Information Integration, 6, 1-10. doi:10.1016/j.jii.2017.04.005.

Masolo, C., Borgo, S., Gangemi, A., Guarino, N. \& Oltramari, A. (2003). Ontology Library. WonderWeb Deliverable D18 (ver. 1.0, 31-12-2003).

Matsokis, A. \& Kiritsis, D. (2010). An ontology-based approach for product lifecycle management. Computers in Industry, 61(8), 787-797. doi:10.1016/j.compind.2010.05.007.

Monostori, L. (2014). Cyber-physical production systems: Roots, expectations and R\&D challenges. Procedia Cirp, 17, 9-13. doi:10.1016/j.procir.2014.03.115.

Mossakowski, T., Codescu, M., Neuhaus, F. \& Kutz, O. (2015). The distributed ontology, modeling and specification language - DOL. In A. Koslow and A. Buchsbaum (Eds.), The Road to Universal Logic. Studies in Universal Logic (pp. 489-520). Cham: Birkhäuser. doi:10.1007/978-3-319-15368-1_21.

Negri, E., Fumagalli, L., Garetti, M. \& Tanca, L. (2016). Requirements and languages for the semantic representation of manufacturing systems. Computers in Industry, 81, 55-66. doi:10.1016/j.compind.2015.10.009.

Otte, N.J., Kiritsis, D., Ali, M.M., Yang, R., Zhang, B., Rudnicki, R., Rai, R. \& Smith, B. (2019). An ontological approach to representing the product life cycle. Applied Ontology, 14(2), 179-197.

Palmer, C., Urwin, E.N., Pinazo-Sánchez, J.M., Cid, F.S., Rodríguez, E.P., Pajkovska-Goceva, S. \& Young, R.I.M. (2016). Reference ontologies to support the development of global production network systems. Computers in Industry, 77, 48-60. doi:10.1016/j.compind.2015.11.002.

Perzylo, A., Somani, N., Rickert, M. \& Knoll, A. (2015). An ontology for CAD data and geometric constraints as a link between product models and semantic robot task descriptions. In Intelligent Robots and Systems (IROS), 2015 IEEE/RSJ International Conference on (pp. 4197-4203). doi:10.1109/IROS.2015.7353971.

Salustri, F.A. (1997). A formal theory for knowledge-based product model representation. In M. Mäntylä, S. Finger and T. Tomiyama (Eds.), Knowledge Intensive CAD. KIC 1996. IFIP - The International Federation for Information Processing (pp. 59-78). Boston, MA: Springer.

Shani, U., Franke, M., Hribernik, K.A. \& Thoben, K.D. (2017). Ontology mediation to rule them all: Managing the plurality in product service systems. In Systems Conference (SysCon), 2017 Annual IEEE International (pp. 1-7). 
Simons, P.M. \& Dement, C.W. (1996). Aspects of the mereology of artifacts. In R. Poli and P. Simons (Eds.), Formal Ontology (pp. 255-276). Dordrecht: Springer. doi:10.1007/978-94-015-8733-4_10.

Stevens, R., Lord, P., Malone, J. \& Matentzoglu, N. (2019). Measuring expert performance at manually classifying domain entities under upper ontology classes. In Web Semantics: Sicence, Services and Agents on the World Wide Web (in press).

Terkaj, W., Pedrielli, G. \& Sacco, M. (2012). Virtual factory data model. In Proceedings of the Workshop on Ontology and Semantic Web for Manufacturing, Graz, Austria (pp. 29-43).

Tessier, S. \& Wang, Y. (2013). Ontology-based feature mapping and verification between CAD systems. Advanced Engineering Informatics, 27(1), 76-92. doi:10.1016/j.aei.2012.11.008.

Uschold, M. \& Grüninger, M. (1996). Ontologies: Principles, methods and applications. The Knowledge Engineering Review, 11(2), 93-136. doi:10.1017/S0269888900007797.

Usman, Z., Young, R.I.M., Chungoora, N., Palmer, C., Case, K. \& Harding, J.A. (2013). Towards a formal manufacturing reference ontology. International Journal of Production Research, 51(22), 6553-6572. doi:10.1080/00207543.2013.801570.

Vegetti, M., Leone, H. \& Henning, G. (2011). PRONTO: An ontology for comprehensive and consistent representation of product information. Engineering Applications of Artificial Intelligence, 24(8), 1305-1327. doi:10.1016/j.engappai.2011. 02.014 . 